\title{
The Impact of Variations in Membrane Capacitance on the Detected Neural-Electronic Signal
}

\author{
Robert B. Szlavik \\ Center for Biomedical Engineering and Rehabilitation Science, Louisiana Tech University, USA .
}

\begin{abstract}
There have been various theoretical and experimental studies presented in the literature that focus on interfacing neurons with discrete electronic devices such as transistors. The demonstrated lack of reproducible fidelity of the nerve cell action potential at the device junction would make it impractical to implement these devices in any neural prosthetic application where reliable detection of the action potential was a pre-requisite. In this study, the impact of typical variations in membrane capacitance on the detected neural signal is investigated.
\end{abstract}

Keywords - Neural-electronics, membrane capacitance

\section{INTRODUCTION}

Specific nerve cell excitation using a semiconductor based electronic device has been demonstrated by Fromherz and Stett [1]. It has also been demonstrated experimentally by Weis and Fromherz, as well as Vassanelli and Fromherz, that a detectable signal can be conducted from a neuron in vitro to a silicon transistor [2;3]. Direct interface and subsequent signal conduction between individual neurons and single transistors would, if practically achievable in an in vivo environment, have the potential of providing a solution to many of the localization issues associated with conventional non-specific field based functional electrical stimulation sensory nerve signal detection approaches.

While some characteristics of the interface region are open to design manipulation, other characteristics, such as the membrane capacitance, are not subject to design modification. Typical variations in these characteristics can have a significant impact on the detected signal at the junction. This simulation study investigates the fidelity of the detected nerve signal for different values of membrane capacitance.

\section{Methodology}

An idealized cylindrical geometry was assumed for the neuron. This geometry was chosen as a simplified approximation of the Retzius cell used in neuron-transistor interface experiments presented by Weis and Fromherz [2].

Although the fundamental physical parameters used in determining the equivalent circuit component values shown in Fig. 1., are specified in a per unit area format, defining the geometric parameters of the neuronal radius $a_{j}$ and length $\boldsymbol{L}$ allow the circuit component values to be specified in absolute units. The physical parameters related to the membrane and the transistor are shown in Table 1., along with the definition of the circuit components.

\section{TABLE I}

List of the variables and physical as well as geometric parameters that were used in the equivalent circuit model.

\begin{tabular}{|c|c|c|}
\hline$\overline{a_{j}}$ & Interface and nerve cell radius & $10(\mu \mathrm{m})$ \\
\hline$d_{j}$ & Nerve cell transistor seal distance & $10(\mathrm{~nm})$ \\
\hline $\boldsymbol{L}$ & Length of the nerve cell & $40(\mu$ \\
\hline$A_{\psi}$ & Free membrane region area & $a_{J}^{2}+2 \pi a_{J} L\left(\mathrm{~cm}^{2}\right)$ \\
\hline$A_{P}$ & Interfaced membrane region area & $\pi a_{j}^{2}\left(\mathrm{~cm}^{2}\right)$ \\
\hline$T$ & Temperature & $6.3\left({ }^{\circ} \mathrm{C}\right)$ \\
\hline$t_{t}$ & Simulation time step & (s) \\
\hline $\boldsymbol{k}$ & Number of time steps & 3000 \\
\hline$\rho_{J}$ & Extracellular environment resistivity & $(\Omega \mathrm{cm})$ \\
\hline$c_{J G}$ & Gate oxide capacitance per unit area & $0.3\left(\mu \mathrm{F} / \mathrm{cm}^{2}\right)$ \\
\hline$c_{\mathrm{Na}}{ }^{0}$ & Extracellular sodium ion concentration & $0.491(\mathrm{~mol} / \mathrm{L})$ \\
\hline$c_{N a}{ }^{i}$ & Intracellular sodium ion concentration & $0.05(\mathrm{~mol} / \mathrm{L})$ \\
\hline$c_{K}^{0}$ & Extracellular potassium ion concentration & $0.02011(\mathrm{~mol} / \mathrm{L})$ \\
\hline$c_{K}^{i}$ & Intracellular potassium ion concentration & $0.400(\mathrm{~mol} / \mathrm{L})$ \\
\hline$G_{N a}{ }^{M}$ & Maximum sodium conductance per unit area & $0.120\left(\mathrm{~S} / \mathrm{cm}^{2}\right)$ \\
\hline$G_{E}{ }^{M}$ & Maximum potassium conductance per unit area & $0.036\left(\mathrm{~S} / \mathrm{cm}^{2}\right.$ \\
\hline$m$ & Hodgkin Huxley first order kinetic variable [4] & \\
\hline $\boldsymbol{h}$ & Hodgkin Huxley first order kinetic variable [4] & \\
\hline $\boldsymbol{n}$ & Hodgkin Huxley first order kinetic variable [4] & \\
\hline$\Psi_{N a}$ & Froe membrane region sodium conductance & \\
\hline$\Psi_{K}$ & Free membrane region potassium conductance & \\
\hline$P_{N a}$ & Interfaced membrane region sodium conductance & \\
\hline$P_{K}$ & Interfaced membrane region potassium conducta & tance \\
\hline $\boldsymbol{R}_{J}$ & Interface junction seal resistance [2] & $\rho_{J} /\left(5 \pi d_{J}\right)(\Omega$ \\
\hline$c_{M}$ & Membrane capacitance per unit area & $\left(\mathrm{F} / \mathrm{cm}^{2}\right)$ \\
\hline$C_{\psi}$ & Free membrane region capacitance & \\
\hline$C_{P}$ & Interfaced membrane region capacitance & \\
\hline$C_{J G}$ & Gate oxide capacitance & $c_{J G} \pi a_{j}^{2}(\mathbf{F}$ \\
\hline$V_{N a}$ & Sodium ionic (Nernst) potential & \\
\hline$V_{R}$ & Potassium ionic (Nernst) potential & \\
\hline$V_{I}$ & Intracellular potential & \\
\hline$V_{J}$ & Interface junction potential & \\
\hline$V_{M}$ & Interface region transmembrane potential & \\
\hline$I$ & Injected stimulus current & (A) \\
\hline
\end{tabular}

The non-linear equivalent circuit that incorporates the non-linear properties of the membrane is shown in Fig. 1. Based on the Weis, Fromherz hypothesis, this model was developed by dividing the membrane into a region that is interfaced with the electronic device, represented by the $P$ labeled circuit components, and a free region of the membrane, represented by the $\Psi$ labeled circuit components as per (1).

$$
\begin{aligned}
& \Psi_{N a}=A_{\Psi} G_{N a}^{M} m^{3} h \\
& \Psi_{K}=A_{\Psi} G_{K}^{M} n^{4} \\
& \mathbf{P}_{N a}=A_{\mathrm{P}} G_{N a}^{M} m^{3} h \\
& \mathbf{P}_{K}=A_{\mathrm{p}} G_{K}^{M} n^{4}
\end{aligned}
$$




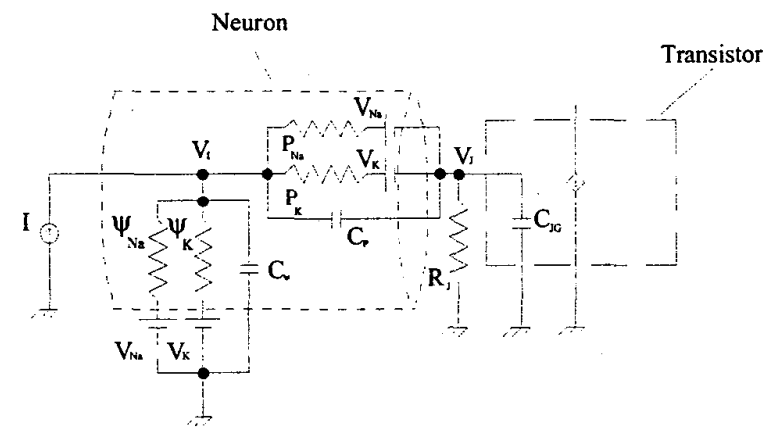

Fig. 1. Non-linear equivalent circuit model of the neuron-transistor configuration. The equivalent circuit component values are calculated using the physical parameters and formulas shown in Table 1 .

The coupled ordinary differential equations (2) implemented in the solver routine were written in terms of the intracellular potential $V_{I}$ and the transmembrane potential $V_{M}$ across the region of the membrane interfaced with the transistor gate.

$$
\begin{aligned}
& \Psi_{N a}\left(V_{N a}-V_{I}\right)+ \Psi_{K}\left(V_{K}-V_{I}\right)-C_{\Psi} \frac{d V_{I}}{d t}+I \\
&+ \mathrm{P}_{N a}\left(V_{N a}-V_{M}\right)+\mathrm{P}_{K}\left(V_{K}-V_{M}\right)-C_{\mathrm{P}} \frac{d V_{M}}{d t}=0 \\
& \mathrm{P}_{N a}\left(V_{M}-V_{N a}\right)+\mathrm{P}_{K}\left(V_{M}-V_{K}\right)+C_{\mathrm{P}} \frac{d V_{M}}{d t}-\frac{V_{I}}{R_{J}} \\
&+\frac{V_{M}}{R_{J}}-C_{J G} \frac{d V_{I}}{d t}+C_{J G} \frac{d V_{M}}{d t}=0
\end{aligned}
$$

A relationship can be defined between the intracellular, junction and transmembrane potentials as per (3).

$$
V_{M}=V_{l}-V_{J}
$$

A Runge-Kutta ordinary differential equation solver was used to calculate the response of this circuit to a brief $10 \mu$ s stimulus current pulse.

The square root of the sum of the squared differences between the intracellular potential $V_{I}$ and the junction potential $V_{J}$ was used to quantify the reliability of the reproduction of the nerve cell action potential at the junction, as per (4), where $t_{i}$ is the time step and $k$ is the number of time steps.

$$
\delta=\sqrt{\sum_{i=0}^{k-1}\left[V_{1}\left(t_{i}\right)-V_{J}\left(t_{i}\right)\right]^{2}}
$$

\section{RESULTS}

The effect of variations in membrane capacitance on the reliability with which the nerve cell action potential was reproduced at the junction was also studied and quantified as per (4) for a physiologically relevant range of membrane capacitance values. A significant improvement in the reliability with which the action potential was reproduced at the junction was observed for larger values of membrane capacitance as per Fig. 2.

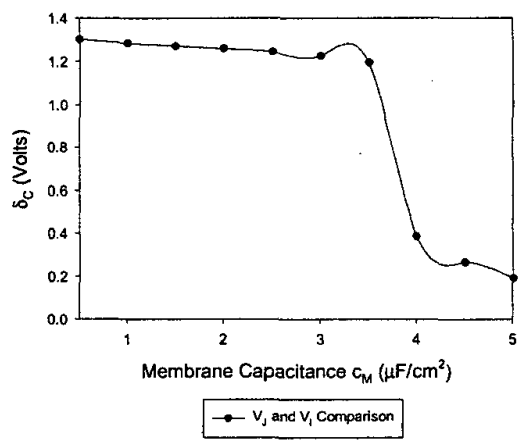

Fig. 2. Norm of the difference between the intracellular potential $V_{I}$ and the junction potential $V_{J}$ as a function of membrane capacitance. This curve quantifies the reliability with which the intracellular action potential is reproduced at the junction. A stimulus of $40 \mathrm{nC} / \mathrm{cm}^{2}$ and an extracellular resistivity of $25 \mathrm{k} \Omega \mathrm{cm}$ were used.

\section{DISCUSSION}

The intracellular action potential waveform is reproduced at the junction without significant distortion when no action potential is initiated in the region of the membrane interfaced with the device. Larger values of membrane capacitance result in smaller initial changes in potential across the cell membrane. Small values of membrane capacitance yield larger initial changes in transmembrane potential that can be sufficient to generate an action potential in the region of the membrane interfaced with the device. For intermediate values of membrane capacitance, an action potential can still be generated in the interface region of the membrane as a result of the action potential generated in the free region of the membrane.

\section{REFERENCES}

[1] Fromherz, P..and Stett, A., "Silicon-neuron junction: capacitive stimulation of an individual neuron on a silicon chip," Physical Review Letters, vol. 75, no. 8, pp. 1670-1673, 1995.

[2] Weis, R. and Fromherz, P., "Frequency dependent signal transfer in neuron transistors," Physical Review E, vol. 55, no. 1, pp. 877889, Jan. 1997

[3] Vassanelli, S. and Fromherz, P., "Transistor records of excitable neurons from rat brain," Applied Physics A, vol. 66 pp. 459-463, 1998.

[4] Weiss, T. F., Cellular Biophysics Cambridge: MIT Press, 1996. 